\title{
Immersive Co-operative Psychological Virtual Environments (ICPVE)
}

\author{
Ronan Jamieson, Adrian Haffegee, and Vassil Alexandrov \\ Advanced Computing and Emerging Technologies Centre, \\ The School of Systems Engineering, University of Reading, \\ Reading, RG6 6AY, United Kingdom \\ $r$.jamieson@reading.ac.uk
}

\begin{abstract}
Virtual Reality (VR) has been used in a variety of forms to assist in the treatment of a wide range of psychological illness. VR can also fulfil the need that psychologists have for safe environments in which to conduct experiments. Currently the main barrier against using this technology is the complexity in developing applications. This paper presents two different co-operative psychological applications which have been developed using a single framework. These applications require different levels of co-operation between the users and clients, ranging from full psychologist involvement to their minimal intervention. This paper will also discuss our approach to developing these different environments and our experiences to date in utilising these environments.
\end{abstract}

\section{Introduction}

Psychologists are constantly seeking new methods and technologies for treating a range of psychological illness. They also have a need to created realistic controllable environments in which to conduct research into the different psychological aspects of human behaviour. One such technology that can fulfil both these requirements is VR, which provides users with the means to create $3 \mathrm{D}$ computer generated virtual environments (VE's). These virtual environments can be viewed in a variety of ways using different display technology from nonimmersive systems (e.g. PC desktops) to semi-immersive (e.g. head mounted displays, HMD's[1]) right to fully-immersive systems (e.g. CAVE[2]). A fully immersive system allows us to be bodily immersed within the virtual environment, this assists in creating a feeling of presence. As mentioned by Slater "presence means that the user constructs a mental spatial model out of virtual stimuli and the perception of the self in the virtual environment" 3]. The advantage that a fully-immersive system gives the therapist/psychologist is, providing the client/user with a increased feeling of presence. This will help lead to a more successful treatment outcome for the client, as the environment will be more involving. Our applications will be focusing on utilising a fully immersive system for these reasons.

There are many different options on what constitutes a co-operative virtual environment, and some of these will be discussed in more detail in the next section. In terms of what is considered a co-operative virtual environment for this

M. Bubak et al. (Eds.): ICCS 2008, Part III, LNCS 5103, pp. 438 445, 2008.
(C) Springer-Verlag Berlin Heidelberg 2008 
paper the following approach has been taken which has had to also consider the terms from a physiological point of view as well. From a psychological viewpoint different types of environments require varying levels of co-operation between client/user and therapist/psychologist. Therefore we have used the term in the following context. A full co-operative environments requires continual feedback from the client to enable the therapist to modify the environment. This modification is required to enable the client to overcome their phobias/fears and bring about a positive treatment outcome. Where as an automatic co-operative environment reduces the needs on the physiologist to continually control the environment as once the user completes a set task then the system can automatically move onto the next defined task, this allows the physiologist to focus on analysing the actions of the user. Also all actions of the user (e.g. head movement, length of time in a particular position/area) is recorded by the system for further off-line analysis. It should be noted that co-operation should not be confused with collaboration, in terms of $\mathrm{VE}$ these are two distinct different approaches.

The following section examines the related work concerning using VR for treating physiological illness and as an experiential tool. It also outlines current research into what is considered a co-operative virtual environments. Section three provides an overview of how we have used our framework to create our different environments and discuss some of the specific requirements that each environment has. This is then followed by an examination of our two application areas that were chosen. Finally our conclusions are presented and future work if required is outlined.

\section{Related Work}

When investigating previous work carried out in this area it becomes apparent that there are two main topics that needed to be considered. The first being the use of VR in the treatment of psychological illness. The second being, what is considered co-operation when using VEs.

The main focus for using VR with treating clients psychological problems has focused on using HMD's to created the virtual environment in which clients are treated. Using this techology the most successfully treated illness have been situational based phobias. This is due to the fact the VR is a powerful medium which allows for the creation of realistic environments. Situational phobias are related to a persistent fear of a particular stimulus and therefore leads to an avoidance of that stimulus. Acrophobia is an example of this type of phobia, it is concerned with the fear of heights. Traditional therapy uses in-vivo techniques to treat this, where as VR has been found to be as if not more successful than this technique 4. With the added benefits of the being able conduct the treatment in the privacy and safety of the therapists office. Also complex and potentially costly situations are possible to recreate virtually. Another area that VR is currently being investigated as a potential therapeutic tool is Post Traumatic Stress Disorder (PTSD) 5]. 
When implementing a co-operative psychological VE it is important to understand what interactions will be required and approaches others have taken. In an traditional VE when dealing with users, who share the same VE and are manipulating the same objects, there are some clear distinctions to be made in the way that they work together. In terms of VE there is a difference between cooperation and collaboration. According to Broll, co-operative implies joint editing of shared objects, while collaborative additionally allows truly concurrent editing [6]. Where as Margery defines co-operation as a situation where two or more people interact on the same object in a concurrent but co-operative way 7 . As our interactions involve both the client/user and therapist/psychologist our version of co-operation is very similar but different, depending on what outcome is required. In our case both parties are jointly engaged in the environment and through their actions (conscious and sub-conscious) act on objects with in the scenario which effects the overall outcome of the session.

\section{System Overview}

Our two different environments have been developed using a single framework, VieGen [8, which is used for the creation and control of Virtual Interactive Environments. It aims to provide users with limited technical ability the power to create their own virtual environments without the need of learning a complex programming language. It also has a API for more advanced users.

A system overview of our approach can be seen in figure 1. This approach allows us to take advantage of the modular nature of VieGen.

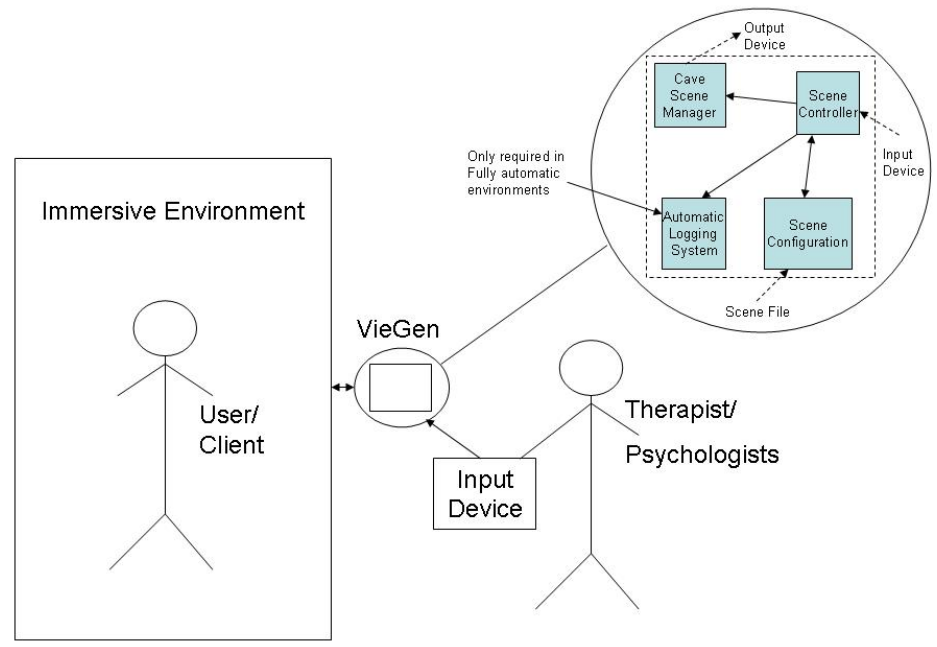

Immersive Co-operative Virtual Environments - System Overview 
The following features of the framework are used in our applications:

\subsection{Scene Configuration}

The Scene Configuration module is responsible for loading the scene file. The scene file is a text file written either in plain text or XML. It contains a description of the objects we require to create the environment, also the position and scale of these objects. We can also assign attributes to objects if we require (e.g. a car object can be dynamic and thus will be able to move around the scene either on a random or pre-set path).

\subsection{Logging}

The Logging Module allows the psychologist to automatically capture data from the experimental sessions. This data can be of various different parameters and date/time stamped. An example of these parameters could be the position the users head was in at a particular location within the environment and for how long they remained in a particular pose. It is a fully configurable module, which allows the psychologist to decide which parameters they require capturing before the commencing of the session.

\subsection{Cave Scene Manager (CSM)}

The Cave Scene Manager Module is used for the internal representation of a user in the VE and manages the basic hierarchy. It provides a generic abstraction layer to the underlying VR hardware allowing system independence. By utilising the tracked users head position and field of view, it manages which objects would be observed and thus require rendering. It controls how this should be achieved and maps the resulting display onto the VR hardware.

\subsection{Scene Controller}

The Scene Controller Module takes care of the maintenance and control of the active environment. It handles the position and movements of any dynamic objects. It looks after the the logical hierarchies between objects, and uses these for grouping their behaviour.

\section{Application Areas}

Creating applications that fulfil the needs of psychologists and therapists has traditionally being an expensive and complex process, involving large amounts of a developers time and effort. This has not always delivered what the end users required. But by using our approach we aim to assist the end users in developing co-operative environments that fulfil their needs, by allowing them to quickly and easily build these environments themselves. 
Two specific applications have been develop which address different psychological needs with varying levels of co-operation. The first application is concerned with addressing the requirements of creating a fully co-operative environment for the treatment of situational phobias. Where as the second application is used to create a automatic co-operative experimental environment for studying aspect of human behaviour. These have been both developed using the same framework, using modules of the VieGen framework that fulfil their requirements.

\subsection{Fully Co-operative Virtual Environment}

As mentioned previously VR has been used for the successful treatment of situational phobias. One such phobia, that has very serious consequences for the clients who suffer from it is, Post Traumatic Stress Disorder (PTSD). This is usually caused by the client being placed in a scenario that causes a traumatic event to occur to that person. This could be a violent assault, terrorist attacks, military combat amongst others. We have based our application on a military combat environment. This application was required to be fully co-operative due to the fact that the client must engage with their therapist. They share the environment in terms of the client and therapist are communicating continually about the environment and situations that occur within the session. They discuss the feelings/emotions that gets invoked during the session. This co-operation must take place to allow the client to move on emotionality/psychologically.

The therapist uses an input devices with contains a GUI, which allows them to control the environment variables (e.g. time of day, audio, movement of people/vehicle). Based on the clients case notes, the therapist will have a record of the traumatic event that lead to the clients PTSD. From this information the therapist can increase the intensity of the environment (e.g. more explosions, insurgents appearing) and guide the client to a successful outcome. This outcome will involve a reduction in the clients PTSD symptoms. The current application is based on a simple environment that allows the client to navigate through an scenario that resembles a downtown market in a middle eastern country (see Figure 2(a)), there are also people and Vehicles within the environment (e.g. soldiers, Humvee). Other features include the ability to add extra audio effects (e.g. gunfire, explosions, sirens), we can also add and remove insurgents (see Figure 2(b)) from the environment.

The environment has been tested on a number of volunteers. Due to the serious nature of the illness, it was not possible to intially test the environment with clients suffering from PTSD. The volunteers time was used to test the immersion of the environment versus a HMD version. Also tested was the interaction with the different elements within the environment (e.g. insurgents being added and remove from the scene). The audio elements are vital to the feeling of presence with the environment and various combinations where tested with the volunteers feeding back on the most realistic audio effects. 

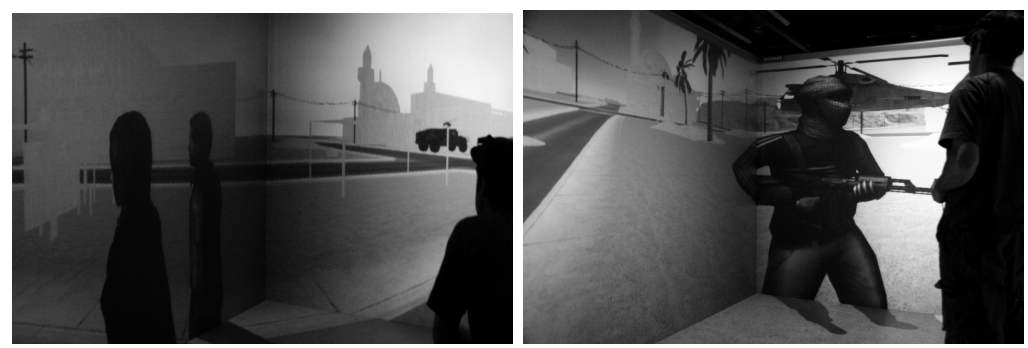

Fig. 1. (a) Market Place (b) Insurgent Appearing

\subsection{Automatic Co-operative Virtual Environment}

The ability to create controllable, measurable and realistic environments is a requirement for psychologists that seek to study different aspect of human behaviour. The developed application was required to be a fully automatic cooperative environment, the user would complete the task set and the system would move onto the following task, thereby the system and user are automatically co-operating with the psychologist. This feature allowed the psychologist to observe and analyse the users behaviour and reactions. The application developed was concerned with customer shopping behaviour, with the aim being to carry out a psychological analysis of whether different layouts of products, both in positioning and in shelf dressing around them, would have any effect on the choice of users' selected products.

Two series of trials of the environment were carried out with a range of different users. The first being a Search Test, this test was to record the movements and time taken for a user attempting to locate a product in a VE. The user started next to one of a number of different shelf layouts. They were shown one or two virtual products when they then had to locate on the shelf. By recording how the user moved with the environment, including their head positions and movements, it was possible to reconstruct the way they acted within the test. The purpose of this test was to discover if there was any relationship between searching and the layouts used. An example of this trail can be seen in Figure 3(a).

The second trail was a Primed Selection Test, this test required the user to move along a fixed path within the environment. Along the path were ten categories of products. The first time along the path the subjects were asked to select specific items from a list, and the second time they were free to choose themselves. The hypothesis being investigated, was whether priming the users with the sample product would cause them to select, or at least notice those items. An example of this trail can be seen in Figure 3(b).

These trials were conducted with 16 users, who completed both tasks. The main points of note that came from these trails were that the effects of the differnet layouts did have an noticeable effect on the users ability to find particular products and that if a user was primed about a certain product they could find it quicker, and were more likely to choose the primed product when given a free choice. 

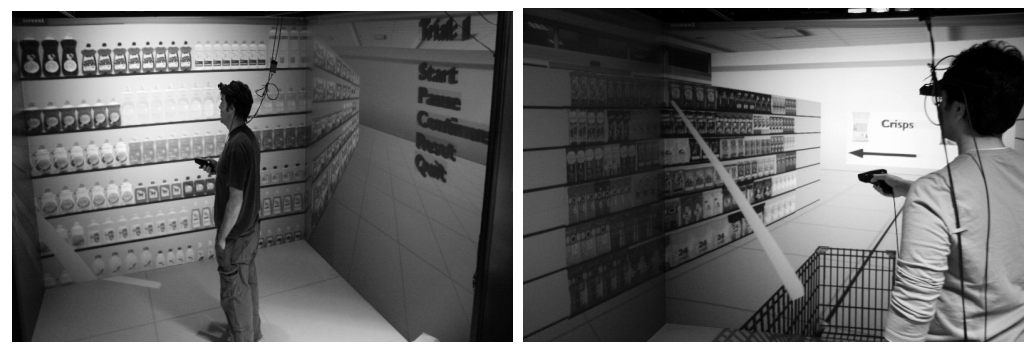

Fig. 2. (a) Search Trail (b) Primed Selection trail

\section{Conclusion and Future Work}

This paper has described the successful development of two different co-operative psychological environments for immersive VR systems. Each application has incorporated the required levels of co-operation that is needed. This ranged from fully co-operative to automatic co-operative environments. These applications are currently at different stages of implementation, the automatic co-operative environment (i.e. customer shopping behaviour) has been utilised in successfully testing various different hypothesis on a wide range of different consumers, where as the fully co-operative environment (i.e. PTSD) is awaiting user trails which should commence shortly.

Future work will include expanding the range and complexity of the consumer trails in the automatic environment. We will also endeavour to increase the cross section of consumers that are to be tested. Automatic environments that incorporate tracking the eye position of the consumer rather than head position will be investigated as this should increase the accuracy of the gaze data captured. Increasing the realism of the fully co-operative environment by the use of haptic feedback will be researched. The range of audio clips for both environments will be expanded which also should increase the feeling of presence within each application. Expanding the range of PTSD environments will be investigated, to try and discover if there is a need for this approach within other professions which are exposed to potentially stressful scenarios (e.g. fire-fighters, police). Research will also be carried out into the possibilities and advantages of enabling the environments to be networked.

\section{References}

1. Sutherland, I.: A head-mounted three-dimensional display. In: Proceedings of Fall Joint Computer Conference, vol. 33, pp. 757-764 (1968)

2. Cruz-Neria, C., et al.: The CAVE: audio visual experience automatic environment. Communication of the ACM 35(6), 64-72 (1992)

3. Slater, M., et al.: Immersion, Presence and Performance in Virtual Environments: An Experiment using Tri-Dimensional Chess. ACM Virtual Reality Software and Technology (1996) 
4. Schuemie, M.J., et al.: Treatment of Acrophobia in Virtual Reality: a Pilot Study. In: Broeckx, F., Pauwels, L. (eds.) Conference Proceedings Euromedia, Antwerp, Belgium, May 8-10, pp. 271-275 (2000)

5. Rizzo, A.A., et al.: User-Centered Design Driven Development of a VR Therapy Application for Iraq War Combat-Related Post Traumatic Stress Disorder. In: Proceedings of the 2006 International Conference on Disability, Virtual Reality and Associated Technology, pp. 113-122 (2006)

6. Broll, W.: Interacting in distributed collaborative virtual environments. In: VRAIS, Los Alamitos, pp. 148-155 (March 1995)

7. Margery, D., et al.: A general framework for cooperative manipulation in virtual environments. In: Gervautz, M., Hildebrand, A., Schmalstieg, D. (eds.) Virtual Environments, Eurographics, pp. 169-178. Springer, Heidelberg (1999)

8. Haffegee, A., et al.: Creation and Control of Interactive Virtual Environments. In: Alexandrov, V.N., van Albada, G.D., Sloot, P.M.A., Dongarra, J. (eds.) ICCS 2006. LNCS, vol. 3992, pp. 595-602. Springer, Heidelberg (2006) 